\title{
CI-UNA: Potenciando el desarrollo profesional del graduado universitario
}

\author{
CI-UNA: Empowering the Professional Development of \\ the University Graduate
}

\author{
Cinthya Olivares Garita \\ Universidad Nacional \\ Heredia, Costa Rica \\ cinthya.olivares.garita@una.ac.cr \\ Cristina Melissa Vargas Barboza \\ Universidad Nacional \\ Heredia, Costa Rica \\ krime0590@gmail.com
}

Recibido: 24/04/2020 Aceptado: 12/08/2020

Resumen. La Universidad Nacional (UNA), una de las cinco universidades públicas más prestigiosas de Costa Rica, "propicia la formación integral del estudiante y su educación permanente, que lo debe preparar para que actúe con responsabilidad ciudadana y contribuya al desarrollo de una sociedad más justa y solidaria" (Universidad Nacional, 2005, p. 5). Uno de los motores de la acción sustantiva de la UNA es la extensión social y la transferencia de conocimiento. A partir del 2010, en el campus de la Universidad Nacional en Pérez Zeledón, se inició el proyecto CI-UNA (Centro de Idiomas de la Universidad Nacional), que brinda una función dual, primeramente, desarrollar competencias en los ciudadanos y las ciudadanas de la localidad mediante la impartición de cursos conversacionales de Inglés, y segundo, ofrecer fuentes de trabajo a estudiantes graduados de la carrera Enseñanza del Inglés de este campus. El presente estudio exploratorio pretende analizar el papel del proyecto CI-UNA como mecanismo clave para la inserción de las personas egresadas de la carrera Bachillerato en la Enseñanza del Inglés en el mercado laboral y la influencia que CI-UNA ejerce en el desarrollo profesional de los profesores instructores. Los principales resultados evidencian el papel preponderante de CI-UNA como proyecto germinador de un 
Revista Universidad en Diálogo • Vol. 10, N. ํ 2, Julio-Diciembre, 2020 • 55-77

ISSN 2215-2849 • EISSN: 2215-4752

URL: https://www.revistas.una.ac.cr/index.php/dialogo/index CORREO ELECTRÓNICO: universidadendialogo@una.ac.cr

DOI: https://doi.org/10.15359/udre.10-2.3

profesorado calificado en áreas como manejo de grupos heterogéneos, desarrollador de actividades propicias para el estudiantado, organización y planeamiento de clases comunicativas; además, este proyecto es reconocido como una gran oportunidad de trabajo, de crecimiento personal y de desarrollo profesional.

Palabras clave: desarrollo profesional, enseñanza del Inglés, extensión social, inserción laboral.

\begin{abstract}
Since 1973, Universidad Nacional (UNA), one of the most prestigious public universities in Costa Rica, "has fostered a holistic formation of the students and permanent education that must prepare them to act as responsible citizens and contribute to developing a more just and caring society" (Universidad Nacional, 2005 , p. 5). One element of substantive action is the social extension and knowledge transfer. From 2010 to present, the Pérez Zeledón Campus implemented a project called CI-UNA (Centro de Idiomas, Universidad Nacional); it has a dual function. First, to develop competencies in the local citizens through teaching conversational English courses; and second, to offer job opportunities to graduate students from the English Teaching Major of this campus. This exploratory study aims to analyze the role of the project CI-UNA as an important mechanism for English Teaching alumni to enter the job market and establish the influence of this project in the instructors' professional growth. The major results evidence the significant role of CI-UNA as a generating project of a qualified group of teachers in areas like heterogeneous group management, development of appropriate activities for the students, and organization and planning of communicative classes. Furthermore, this project is recognized as a great job opportunity, personal growth, and professional development.
\end{abstract}

Keywords: professional development, English teaching, social extension, labor inclusion.

\title{
Introducción
}

La Universidad Nacional (UNA) es una institución de educación superior de alto renombre, fundada en los años sesenta y reconocida como Universidad Nacional de Costa Rica a partir del año 1973. Esta institución se ha posicionado como una universidad líder en la preparación de educadores y educadoras a lo largo y ancho de todo el país desde entonces. Es una universidad estatal que en la actualidad cuenta con tres sedes regionales, seis campus universitarios, una sección regional y una sede interuniversitaria. Dentro de su oferta académica destaca la enseñanza de varias especializaciones, como la enseñanza de diversos idiomas (Español, Francés, Inglés), de la Educación Física, la Religión, la Informática, las Ciencias, las Matemáticas, entre otras. 
URL: https://www.revistas.una.ac.cr/index.php/dialogo/index

CORREO ELECTRÓNICO: universidadendialogo@una.ac.cr

DOI: https://doi.org/10.15359/udre.10-2.3

Además de ser una universidad que se reconoce por su calidad en la formación de profesionales de la educación y en otras áreas de especialización, la acción sustantiva de esta institución de estudios superiores está también basada en la investigación, la producción, la gestión y la extensión (Estatuto Orgánico, 2015, p. 14). Este último elemento ha sido clave en la difusión del conocimiento, la innovación y la actualización a otros estratos de la sociedad. En este particular, las sedes han cumplido un papel preponderante para posicionar a esta universidad pública y lograr su reconocimiento en todo el territorio nacional. Es a través de proyectos de extensión y transferencia del conocimiento que esta casa del saber abre sus puertas a la comunidad local y a la nacional para garantizar el aprendizaje de diferentes competencias. En el caso específico de la enseñanza del Inglés, la Sede Regional Brunca ha puesto en marcha un proyecto de transferencia del conocimiento desde el 2010, denominado Centro de Idiomas, Universidad Nacional (CI-UNA). Este proyecto, además de cumplir con una función social al preparar a los ciudadanos con herramientas para enfrentar la competitividad, ha servido como base para que recién graduados de la carrera Enseñanza del Inglés se incorporen rápidamente al mercado laboral.

Actualmente, el $100 \%$ del personal docente de CI-UNA son graduados de la carrera Enseñanza del Inglés del Campus Pérez Zeledón. Gracias a este fenómeno se inicia esta investigación exploratoria que pretende indagar cómo contribuye CI-UNA en la inserción laboral de los graduados de la carrera Enseñanza del Inglés de la Universidad Nacional, Sede Regional Brunca, Campus Pérez Zeledón.

La población está constituida por una muestra de catorce profesores y profesoras que laboraron para CI-UNA en el 2016. Para el muestreo se tomó en cuenta que CI-UNA fuera la primera o una de las primeras experiencias laborales de estos profesores. De esos docentes, doce continúan trabajando para esta institución y corresponden al $63,15 \%$ de su personal. Los instrumentos utilizados fueron un cuestionario para profesores, una entrevista para las coordinadoras, un cuestionario para otros empleadores y la recolección de evidencias. El cuestionario pretendía medir variables como la percepción de los profesores sobre CI-UNA, los beneficios obtenidos al trabajar ahí, su crecimiento profesional después de esta inserción laboral, entre otras. La entrevista tomaba en cuenta variables como: el propósito de CI-UNA, los beneficios adquiridos por el personal, el acompañamiento brindado a los profesores en esa inserción laboral, entre otras. 
Las evidencias consistieron en recoger evaluaciones aplicadas a profesores durante cada ciclo lectivo para medir su crecimiento profesional. Las evaluaciones fueron ejecutadas por estudiantes de los cursos y una desarrolladora del proyecto. Con estos instrumentos se pretendía obtener la opinión de encuestados y entrevistados, así como la recolección de evidencias tangibles del crecimiento profesional de los profesores de este estudio para brindar objetividad y confiabilidad a este proceso. En el análisis se utilizaron gráficos y figuras para brindar un mejor entendimiento de los resultados y dar confiabilidad al estudio por medio de la triangulación de la información. Dentro de las conclusiones más destacadas sobresalen que el $100 \%$ de los profesores reconocen que trabajar en CI-UNA ha mejorado su práctica docente. Además, los profesores expusieron que trabajar para este centro ha contribuido en su inserción laboral, pues les ha ayudado a ampliar su experiencia, saber manejar distintos tipos de grupos, desarrollar nuevos recursos didácticos, mejorar sus habilidades lingüísticas, entre otros aspectos.

Para el siguiente estudio se establecieron las siguientes preguntas conductoras:

1. ¿Cuál es el papel que cumple el proyecto CI-UNA en la inserción laboral de los egresados de la carrera Bachillerato en la Enseñanza del Inglés de la UNA, SRB, Campus Pérez Zeledón?

2. ¿Cuáles son los beneficios tangibles que obtienen los egresados que laboran para el proyecto CI-UNA?

3. ¿Cuál es el avance en el desempeño profesional de los egresados al impartir los cursos asignados del programa CI-UNA?

4. ¿Qué recomendaciones se pueden sugerir a las desarrolladoras del proyecto CI-UNA para fortalecer el papel que cumple con los recién graduados?

\section{Marco teórico}

\section{Universidad Nacional: la universidad necesaria}

Alrededor de la segunda mitad del siglo veinte, la Universidad Nacional se estableció como la Escuela Normal, y posteriormente, como la Escuela Normal Superior en 1960. Para el año 1973 obtiene el rango de Universidad Nacional, constituyéndose como la segunda universidad pública en ese entonces (UNA institución para Costa Rica, p. 2). Su función social ha favorecido 
URL: https://www.revistas.una.ac.cr/index.php/dialogo/index

CORREO ELECTRÓNICO: universidadendialogo@una.ac.cr

DOI: https://doi.org/10.15359/udre.10-2.3

desde esa fecha hasta la actualidad a gran parte de la ciudadanía nacional, primordialmente a aquellas personas que optan por estudios superiores y que poseen una situación económica desfavorable. De aquí se desprende el lema "la Universidad Nacional, la universidad necesaria".

Para dar una amplia cobertura nacional, la UNA se ha establecido en diferentes puntos del país en tres diferentes sedes regionales (Central, Brunca y Chorotega), las cuales se subdividen en seis campus (Omar Dengo, Benjamín Núñez, Pérez Zeledón, Coto, Liberia y Nicoya), una sección regional (Huetar Norte y del Caribe) y una sede interuniversitaria (Alajuela). Es así como este centro de estudios superiores ha logrado posicionarse a nivel nacional en un puesto de privilegio dentro de las universidades nacionales, ubicándose en el puesto 2, en el puesto 55 a nivel de Latinoamérica y en el puesto 701 a nivel mundial (QS Worldwide university rankings, 2015-2016).

Esta amplia trayectoria de la UNA ha beneficiado la difusión del conocimiento, la investigación, la innovación y la producción en diversos campos de estudio de las ciencias sociales y las ciencias exactas. Principalmente, este centro de estudios ha obtenido un alto prestigio nacional e internacional en la formación de profesionales de la educación. Asimismo, estos profesionales comprometidos formadores de ciudadanos competentes han ensanchado la visión de la universidad a través del desarrollo de proyectos de extensión y transferencia del conocimiento cuyo cometido es favorecer la educación de la comunidad local.

\section{Modelo pedagógico}

El papel de la Universidad Nacional ha sido preponderante en la formación del ciudadano costarricense que se pretende moldear. El siglo XXI ha determinado la adquisición de múltiples competencias y habilidades necesarias para enfrentar sus retos. Estas habilidades se agrupan en tres dimensiones: información, comunicación, ética e impacto social (OCDE, 2010, p. 7). Ante una sociedad tan exigente, la educación no se puede volver insensible hacia el cumplimiento del papel que le corresponde en relación con la formación de la ciudadanía de la Costa Rica del siglo XXI. En virtud de ello, la Universidad Nacional, dentro de su accionar, ha establecido cuidadosamente las pautas para cumplir con esa función protagónica y contribuir al desarrollo de un ciudadano competente en el siglo vigente. A través de su modelo pedagógico, la academia se asegura la formación de ciudadanos, tal y como se plantea en el siguiente extracto del modelo pedagógico: 
Docentes y estudiantes son los protagonistas de los procesos de enseñanza y de aprendizaje, de su innovación y su actualización permanentes. Ambos construyen, en el marco de una relación dialógica permanente, espacios que favorecen el desarrollo del conocimiento y su desarrollo integral como personas, en estrecha relación con las distintas áreas académicas institucionales y el contexto socio-histórico nacional e internacional. (Preámbulo Reglamento general sobre los procesos de enseñanza y aprendizaje de la Universidad Nacional, 2006, p. 2)

Es una misión concreta de esta universidad pública favorecer no solo la excelencia en la preparación académica, sino también el desarrollo profesional de los egresados una vez que dejan las aulas de este centro del saber, a través de la implementación de diferentes mecanismos; esto es lo que encierra un desarrollo integral del estudiantado de la Universidad Nacional.

\section{Expansión de la enseñanza y aprendizaje del idioma Inglés en la Región Brunca: el papel de la Universidad Nacional de Costa Rica}

La Región Brunca contempla cinco cantones de la provincia de Puntarenas (Osa, Corredores, Buenos Aires, San Vito y Golfito) y uno de la provincia de San José (Pérez Zeledón) (Arce, 2006). Alrededor de los años noventa, paralelo al auge del turismo en esta región del país, hubo un interés de los pobladores por aprender las destrezas necesarias para comunicarse en el idioma inglés y poder así establecer relaciones comerciales con los turistas; por esta razón, algunos optaron por enseñar el idioma también. La expansión de la enseñanza de este idioma en esta zona provocó un aumento del número de matrícula en los centros de estudio tanto públicos como privados que ofertaban cursos de Inglés en el año 2012 (Brenes, 2012, p. 14). Igualmente, las universidades públicas instauradas en la Región Brunca, como la Universidad Nacional de Costa Rica y la Universidad Estatal a Distancia, diagnosticaron la demanda del idioma inglés y la incluyeron en su oferta académica a partir de ahí.

La Sede Regional Brunca cuenta con dos campus universitarios: Campus Pérez Zeledón y Campus Coto. En el año 1997, en el Campus Pérez Zeledón se ofertó la carrera Bachillerato en la Enseñanza del Inglés por primera vez, y posteriormente, en el año 2009 la carrera Diplomado en Inglés. ${ }^{1}$ Además, se han ofertado otras carreras vinculadas de algún modo con el aprendizaje competitivo del idioma inglés, tales como el Bachillerato en Gestión

Información tomada de la base de datos de la Coordinación del Área de Idiomas Extranjeros, UNA, SRB, Campus Pérez Zeledón, facilitada por la coordinadora del área 
URL: https://www.revistas.una.ac.cr/index.php/dialogo/index

CORREO ELECTRÓNICO: universidadendialogo@una.ac.cr

DOI: https://doi.org/10.15359/udre.10-2.3

Empresarial del Turismo Sostenible y la Licenciatura en Lingüística Aplicada del Inglés. En el Campus Coto se ofertó el Bachillerato en la Enseñanza del Inglés a partir del año 2011 y el Bachillerato en Gestión Empresarial del Turismo Sostenible en el año 2013. Estas carreras marcaron parte del compromiso de la Universidad Nacional con la comunidad local de proveer las herramientas necesarias para potencializarlos como ciudadanos claves ante los retos del siglo XXI.

Por otra parte, la Universidad Nacional ha expandido su compromiso al desarrollar proyectos que involucren otros estratos de la población. Recientemente, en el año 2010, se inició el proyecto Centro de Idiomas, Universidad Nacional (CI-UNA), en el Campus Pérez Zeledón. A partir de esta fecha y hasta el 2016, el proyecto preparó alrededor de 145 personas que se disponen a aprender inglés y concluir, a lo largo de dos años, con un nivel intermedio avanzado, según el Marco Común Europeo de Referencia para las Lenguas (MCERL).

En el año 2014, CI-UNA, junto con el Ministerio de Educación Pública (MEP) y la Embajada de los Estados Unidos de América, firma un convenio llamado CI-UNA Access Microscholarship Program para otorgar becas a estudiantes de secundaria con una condición económica desventajosa, pero académicamente sobresalientes. En el año 2014 se becaron ochenta estudiantes de diferentes colegios públicos de la zona, quienes cursaron por dos años los diez módulos establecidos de CI-UNA. En el año 2016, se inició otra promoción, esta vez con cien estudiantes becados. El objetivo de este programa es mejorar las habilidades lingüísticas del idioma inglés del estudiantado de edades entre los trece y los dieciséis años, y, asimismo, contribuir con la posibilidad de que tengan una pronta inserción en el mercado laboral, una vez que finalicen sus estudios de secundaria o vincularlos a opciones de estudio en el extranjero. En el año 2015, un grupo de profesionales de la UNA, SRB, inicia un proyecto de regionalización en el cual se incluían cursos de Inglés a comunidades de alto riesgo social por un período de un año. El proyecto se encuentra vigente hasta la actualidad y ha impactado diferentes zonas de la región sur de Costa Rica.

\section{CI-UNA: Brindando proyección UNA a la comunidad}

A partir del año 2010, y a raíz del compromiso de la Universidad Nacional de dar respuesta a las necesidades de la comunidad y las demandas del mercado laboral, se empieza a gestar el proyecto denominado CI-UNA (Centro de Idiomas, Universidad Nacional) en la Sede Regional Brunca, Campus Pérez Zele- 
Revista Universidad en Diálogo • Vol. 10, N. ํ 2, Julio-Diciembre, 2020 • 55-77

ISSN 2215-2849 • EISSN: 2215-4752

URL: https://www.revistas.una.ac.cr/index.php/dialogo/index CORREO ELECTRÓNICO: universidadendialogo@una.ac.cr

DOI: https://doi.org/10.15359/udre.10-2.3

dón. Cuatro académicas de este campus universitario son quienes ejecutan la idea y después de un arduo período de diagnóstico, preparación de programas, metodologías, bibliografía y la creación de un reglamento, se disponen a ofertar los primeros cursos de idiomas, especialmente de Inglés. Los cursos son dirigidos a aquellas personas de trece años en adelante que desean mejorar las habilidades lingüísticas del inglés que ya poseen o aprenderlo por primera vez. Debido a que el proyecto está fundamentado por la misma filosofía humanista de la UNA, los cursos se ofertan a un precio accesible para la población meta.

Los cursos ofertados se programan en once módulos para aquellos que inician desde el curso CI-UNA Principiante (Starter), cursados en modalidad bimestral durante un período de dos años y dos meses. Igualmente, el proyecto ofrece la posibilidad de realizar un examen de ubicación que integra las cuatro habilidades lingüísticas para aquellos que ya poseen algún conocimiento anterior, en este caso específico del inglés. Los cursos han sido programados de manera que se cumplan a cabalidad los niveles de competencia lingüística establecidos por el MCERL. El siguiente cuadro evidencia la distribución de los cursos en relación con las bandas del MCERL, la bibliografía empleada, así como también las horas cursadas durante la duración del programa.

Tabla 1

Vistazo general de estructura de los cursos CI-UNA

\begin{tabular}{|c|c|c|c|c|c|c|c|c|c|c|c|}
\hline \multirow{3}{*}{$\begin{array}{l}\text { Niveles del Marco Común } \\
\text { Europeo de Referencia de } \\
\text { las Lenguas (MCERL) } \\
\text { NIVELES CI-UNA }\end{array}$} & \multicolumn{3}{|c|}{ A1 } & \multicolumn{2}{|c|}{ A2 } & \multicolumn{2}{|c|}{ B1 } & \multicolumn{2}{|c|}{ B1 } & \multicolumn{2}{|c|}{ B2 } \\
\hline & \multicolumn{5}{|c|}{ Usuario básico } & \multicolumn{6}{|c|}{ Usuario independiente } \\
\hline & PRINCIPIANTE & I & ॥ & III & IV & v & vi & VII & VIII & IX & $x$ \\
\hline $\begin{array}{l}\text { Libro de texto American } \\
\text { English File }\end{array}$ & AEF STARTER & AEF $1 \mathrm{~A}$ & AEF $1 \mathrm{~B}$ & AEF 2A & AEF $2 B$ & AEF $3 A$ & AEF 3B & AEF 4A & AEF $4 B$ & CONVERSACIONAL & CONVERSACIONAL \\
\hline Horas & 40 & 40 & 40 & 40 & 40 & 40 & 40 & 40 & 40 & 40 & 40 \\
\hline $\begin{array}{l}\text { Certificado de cumplimiento de } \\
\text { acuerdo a horas de instrucción }\end{array}$ & 40 horas & & & 200 horas & & & & & & 200 horas & \\
\hline
\end{tabular}

Nota: Vistazo general de los niveles lingüísticos del MCERL, niveles de los cursos de CI-UNA, libro de texto AEF, horas de instrucción y aspectos del certificado. Adaptado de Jiménez, Y., Olivares, C. y Palacios, S. (2016).

El objetivo primordial de CI-UNA es preparar al estudiantado para que al concluir el programa finalicen con un nivel de usuario independiente, es decir, un B2. De acuerdo con el MCERL (2002). 
URL: https://www.revistas.una.ac.cr/index.php/dialogo/index

CORREO ELECTRÓNICO: universidadendialogo@una.ac.cr

DOI: https://doi.org/10.15359/udre.10-2.3

Comprende las ideas principales de textos sobre temas concretos y abstractos, incluyendo discusiones técnicas de su especialidad. Es capaz de participar en una conversación con bastante fluidez y sin dificultad; esto le faculta para hablar con nativos sin crear tensión. Produce textos claros y exactos sobre muchos temas y es capaz de expresar su punto de vista acerca de un tema, explicando las ventajas y desventajas de los otros puntos de vista.

Un factor de gran transcendencia para lograr el éxito del programa y el óptimo desarrollo del nivel lingüístico de los estudiantes ha sido el currículo diseñado y el dominio que los instructores tienen del mismo. Para ello, fue necesario trazar un plan para determinar aspectos esenciales de la organización de los aprendizajes que se esperaban lograr en los estudiantes meta. La organización consistía en los siguientes elementos de acuerdo con Brown (1995):

Recolección de información que servirá de base para satisfacer las necesidades de aprendizaje de un grupo de estudiantes. Una vez identificadas estas necesidades, se estiman los logros y objetivos que servirán como las bases para el desarrollo de exámenes, material de enseñanza, actividades y estrategias de aprendizaje (p. 35).

La efectividad del currículo no se detiene con un simple diagnóstico o un plan en el papel: por el contrario, se alimenta de otros elementos que son piezas fundamentales, por ejemplo, la bibliografía, el equipo tecnológico y audiovisual disponible, pero sobretodo el talento humano. Definitivamente, este último elemento es determinante para la sostenibilidad de CI-UNA en el tiempo en cuanto a la calidad de los procesos de enseñanza y aprendizaje.

Para lograr un nivel satisfactorio en los estudiantes, los instructores que conforman la planta de profesores de CI-UNA reciben capacitaciones, supervisión constante $\mathrm{y}$, además, entrenamiento sobre el uso de la metodología denominada instrucción por tareas o TBI (Task-based Instruction), especialmente tareas pedagógicas o realizadas en el aula. De acuerdo con Nunan (2004), una tarea pedagógica es un elemento del trabajo de clase que involucra a los estudiantes en la comprensión, manipulación, producción e interacción en el idioma meta, mientras su atención se enfoca en movilizar el conocimiento gramatical para expresar un significado, y en el cual la intención es expresar un significado más que manipular una forma gramatical. La tarea o actividad debe tener sentido completo, siendo capaz de permanecer por sí misma como un acto comunicativo con un inicio, un intermedio y un fin (p. 4). 
Revista Universidad en DiÁlogo • Vol. 10, N. ํ 2, Julio-Diciembre, 2020 • 55-77

ISSN 2215-2849 • EISSN: 2215-4752

URL: https://www.revistas.una.ac.cr/index.php/dialogo/index CorReo eleCtrónico: universidadendialogo@una.ac.cr

DOI: https://doi.org/10.15359/udre.10-2.3

Dentro del planeamiento de lección que los instructores realizan existen una serie de actividades menores que conllevan a alcanzar una tarea principal o actividad de mayor fuerza y que los aproxima a situaciones de la realidad.

Los instructores cumplen con un formato definido para el planeamiento de sus clases denominado STAR. Cada una de las letras de la palabra STAR representa en inglés una etapa valiosa del proceso de aprendizaje. La "S" significa starter o inicio de clase. Se sugiere al instructor utilizar al menos los primeros diez minutos de la clase para realizar una actividad de motivación que despierte en los estudiantes el deseo de permanecer en el aula por dos horas y media (durante las clases vespertinas entre semana) o cinco horas (durante las clases de los días sábados) y mejorar la receptividad hacia el aprendizaje nuevo y fresco. Basado en los argumentos planteados por Peacock (1997), los warm-ups o inicios de clases aumentan la motivación en los estudiantes. Asimismo, el autor señala que la "motivación es el interés de los estudiantes y el entusiasmo por las tareas y los materiales del aprendizaje utilizados en la clase, y ésta indica los niveles de atención, concentración y disfrute" (p. 145). A la luz de esta idea es que se sugiere a todos los instructores dedicar al menos de diez a quince minutos para preparar a los estudiantes.

$\mathrm{La}$ "T" significa tackle, que se define como la etapa de "acercamiento" a la gramática, al vocabulario, a la pronunciación y al deletreo en el idioma, entre otros aspectos. Es una etapa que requiere mucha preparación de parte del instructor para desarrollar explicaciones claras y correctas de los temas de estudio. Además, esta etapa involucra mucha creatividad y capacidad de los instructores para capturar la atención de los estudiantes y generarles interés hacia los contenidos.

La letra "A" significa automatization o automatización. Esta etapa es la denominada central o core activity. Es la etapa más importante, ya que los estudiantes demuestran el conocimiento adquirido durante la lección a través del desarrollo de un debate, una mesa redonda, un panel, una entrevista o cualquier actividad que se asemeje a un evento de la realidad, tales como la representación de una conversación en un restaurante, depositar un cheque, comprar en el supermercado, una visita al médico, etc. Es en esta etapa donde el estudiante produce el lenguaje que el instructor determinó en el plan de lección.

La letra "R" significa recycling o reciclaje, lo cual, para efectos de la lección que se imparte en CI-UNA, es visto como una evaluación formativa o un espacio de retroalimentación (assessment). Al llegar a esta fase, los instructores han recolectado ejemplos de estructuras lingüísticas que los estudiantes no dominaron durante la fase "A". Para esto, el instructor ha sido comunicado sobre la importancia del monitoreo en la etapa en la que el estudiante produce 
URL: https://www.revistas.una.ac.cr/index.php/dialogo/index

CORREO ELECTRÓNICO: universidadendialogo@una.ac.cr

DOI: https://doi.org/10.15359/udre.10-2.3

y el profesor se convierte solamente en un facilitador y un testigo. De acuerdo con Frank (2012):

La evaluación formativa [assessment] debería ser vista como un medio para ayudar a guiar a los estudiantes en su camino hacia el aprendizaje. Ningún procedimiento satisface las necesidades de todos los estudiantes y situaciones, es por esto que necesitamos recordar incorporar una variedad de herramientas para ayudar a que los estudiantes sepan cómo están progresando y cómo redirigir la efectividad de las metodologías y materiales. (p. 32)

En virtud del planteamiento anterior es que los instructores deciden de qué manera repasar las estructuras gramaticales construidas inapropiadamente por los estudiantes. Dentro de las modalidades de retroalimentación que se solicitan están las evaluaciones realizadas por un compañero de clase (peer assessment), las que el mismo estudiante realiza sobre su desempeño (self assessment) y las dirigidas por el instructor (teacher assessment). Para llevar a cabo todas estas modalidades, el instructor desarrolla diferentes técnicas o actividades sobre las cuales requiere una capacitación previa.

\section{CI-UNA con un propósito dual}

CI-UNA exitosamente egresó 145 estudiantes desde el 2010 hasta el 2016 inclusive. $^{2}$ Dichos estudiantes pudieron finalizar el proceso de dos años consecutivos con un desempeño lingüístico ubicado dentro de la banda $\mathrm{B}$, según el MCERL. Este es el primer propósito del programa, la preparación de personas de la comunidad en el manejo de las habilidades lingüísticas de un segundo idioma, en este caso del inglés, para asegurarse una inserción laboral exitosa.

Por otro lado, aunque no ha sido un objetivo concreto e inicial del proyecto, CI-UNA se ha posicionado con el pasar del tiempo como el puente que vincula al estudiante egresado de la carrera Bachillerato en la Enseñanza del Inglés (BEI) con la realidad del mundo laboral y profesional. Por lo general, la mayoría de los instructores del proyecto han sido egresados de la carrera BEI con muy poca o ninguna experiencia profesional. La oportunidad que CIUNA ofrece a estos instructores es única; primeramente, aunque la experiencia sea casi nula, se confía en el sello UNA que los instructores poseen. Esto, al menos, proporciona una garantía del desempeño futuro que los egresados seleccionados tendrán como profesores, ya que se reclutan como instructores del proyecto a aquellos que han sido estudiantes sobresalientes.

Base de datos CI-UNA 
Como parte del desarrollo efectivo de los módulos de los cursos, el instructor de CI-UNA debe superar distintos desafíos profesionales, los cuales se pueden agrupar en tres grandes estadios: atributos personales, responsabilidades y deberes del proyecto y la realidad de la clase (ver figura 1).

El enfrentamiento de dichos desafíos produce en el instructor novato de CI-UNA un enriquecimiento profesional y un posicionamiento en su campo a gran escala. El primer estadio lo constituyen los atributos personales del instructor. Desde el primer instante, durante la selección de los instructores, este aspecto ejerce bastante influencia. El profesor de CI-UNA se destaca por su personalidad amena, humanista, dinámica, creativa y proactiva. Además, la personalidad de los instructores debe crear un ambiente de compenetración (rapport) con los estudiantes. Asimismo, el estilo de enseñanza que se pretende establecer en el desarrollo de los cursos debe ser compatible con la personalidad de los instructores. Es decir, se busca establecer un estilo de enseñanza activo, por lo tanto, el instructor debe ser dinámico y creativo. De lo contrario, se perdería el atractivo del programa que se le atribuye a la personalidad y al estilo de enseñanza de los instructores, así como también a la calidad de los cursos y al precio de costo que los estudiantes pagan por bimestre.

Figura 1. Principales desafíos que enfrentan los instructores

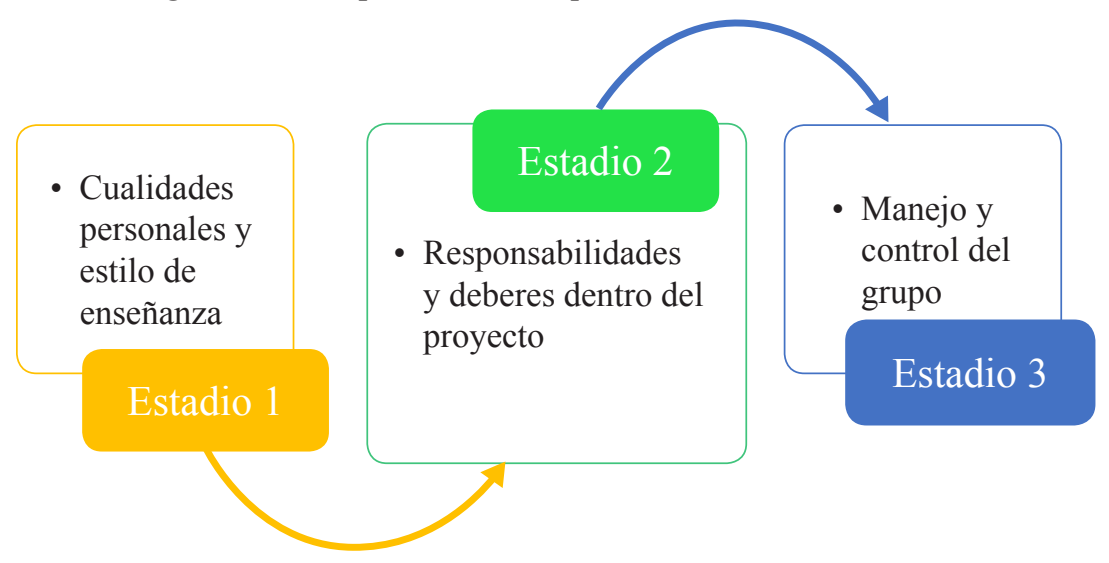

Nota: Elaboración propia. 
URL: https://www.revistas.una.ac.cr/index.php/dialogo/index

CORREO ELECTRÓNICO: universidadendialogo@una.ac.cr

DOI: https://doi.org/10.15359/udre.10-2.3

Dentro de las responsabilidades que el proyecto CI-UNA asigna a sus profesores instructores se encuentra el diseño de un planeamiento de clase por sesión, el diseño de pruebas cortas de comprensión auditiva, gramática y escritura, el control exhaustivo de la asistencia y la participación por clase, la llegada puntual a los cursos, la autoevaluación de su desempeño cada bimestre, el cumplimiento de al menos una hora de atención a los estudiantes y la asistencia a reuniones organizadas por las desarrolladoras. Estas responsabilidades son, de cierto modo, vinculantes en el desarrollo profesional de los profesores que recién inician sus pasos en la enseñanza del Inglés. Para la realización de estas actividades, una de las desarrolladoras ofrece el acompañamiento (mentoring), e igualmente, una vez en el bimestre, se realizan reuniones donde se ofrece un espacio para llevar a cabo un encuentro e intercambio de ideas y resolución de conflictos.

Por otro lado, el manejo y control de los grupos al impartir clases genera en los instructores grandes desafíos. Los instructores "novatos" requieren estrategias efectivas para aminorar el impacto de grupos indisciplinados, el incumplimiento de reglas, la falta de estudio y, sobretodo, para el mejoramiento de la participación efectiva. Para este cometido, una de las desarrolladoras se encarga de dar seguimiento y acompañamiento a los procesos áulicos a través de la observación de clase. Se utiliza para este fin un instrumento de evaluación, el cual es devuelto en físico o digital a los instructores una vez finalizada la observación. El propósito es brindar retroalimentación significativa a los instructores acerca de las fortalezas y las debilidades que se evidenciaron.

\section{Metodología de la investigación}

En esta sección, se presenta la metodología utilizada para la recolección de información necesaria para dar respuesta a las preguntas de investigación establecidas anteriormente. Estas preguntas buscaban establecer cómo CIUNA contribuye en la inmersión laboral de los estudiantes graduados de la carrera Enseñanza del Inglés de la Universidad Nacional, Sede Regional Brunca, Campus Pérez Zeledón. Además, en esta sección se establece el enfoque y el tipo de investigación y se describen los instrumentos utilizados en la recolección de datos. 


\section{Enfoque y tipo de investigación}

El enfoque utilizado en esta investigación es de naturaleza cualitativa, ya que las investigadoras buscan interpretar el fenómeno estudiado en el mismo contexto en donde se desarrolla. De acuerdo con lo expuesto por Denzin y Lincoln (citados por Gall, Gall \& Borg, 2003), la investigación cualitativa estudia cómo ocurren las cosas en su ambiente natural, tratando de darles sentido, o interpretar el fenómeno estudiado según el significado que las personas le dan (p. 24). De igual forma, el tipo de investigación escogido por las investigadoras para este estudio es exploratorio, el cual, según Robson, es una valiosa forma de darse cuenta de lo que está pasando en un lugar determinado, para cuestionar y evaluar un fenómeno desde otro punto de vista (citado por Saunders, Lewis \& Thornhill, 2007, p. 139). Es por eso que, por su naturaleza exploratoria, este tipo de investigación no busca ofrecer soluciones a un problema determinado.

\section{Descripción de los instrumentos}

Para recolectar la información necesaria para el análisis de esta investigación exploratoria cuatro instrumentos fueron aplicados a los participantes de este estudio. Estos instrumentos son: un cuestionario para los profesores de CIUNA que tuvieron a esta institución como su primera o una de sus primeras experiencias laborales, un cuestionario a una de las desarrolladoras del proyecto, una recolección de evidencias y un cuestionario para los nuevos empleadores de algunos de los profesores participantes. Cada uno de los instrumentos es explicado a continuación.

\section{Cuestionario para profesores de CI-UNA}

Este instrumento tuvo por objetivo la recolección de la información relacionada con la percepción de los profesores de Inglés graduados de la carrera Bachillerato en la Enseñanza del Inglés impartido en la Universidad Nacional, Sede Regional Brunca, Campus Pérez Zeledón, que tuvieron su primera o una de sus primeras experiencias laborales en CI-UNA acerca de los beneficios adquiridos y los cambios vistos en su práctica docente luego de trabajar en esta institución. El instrumento se dividió en dos partes. A través de la primera parte se recolectó la información general de los encuestados, como el sexo, la edad, el tiempo laborado para CI-UNA, y otras instituciones donde estén laborando. La segunda parte constaba de siete preguntas abiertas a través de las cuales se recolectó la percepción de los profesores sobre CI-UNA, los 
URL: https://www.revistas.una.ac.cr/index.php/dialogo/index

CORREO ELECTRÓNICO: universidadendialogo@una.ac.cr

DOI: https://doi.org/10.15359/udre.10-2.3

beneficios adquiridos al trabajar ahí, su crecimiento profesional, los retos encontrados y superados, entre otros aspectos.

\section{Cuestionario para la desarrolladora de CI-UNA}

El objetivo de este instrumento consistía en recolectar información relacionada con el proyecto Centro de Idiomas Universidad Nacional (CI-UNA) para determinar cómo este ha influido en la inserción laboral de los graduados de la carrera Bachillerato en la Enseñanza del Inglés de la Universidad Nacional, Sede Regional Brunca, Campus Pérez Zeledón, desde el punto de vista de una de las desarrolladoras del proyecto. El instrumento estaba constituido por trece preguntas abiertas sobre la percepción de una de las desarrolladoras en relación con aspectos como el propósito de CI-UNA, los beneficios adquiridos por el personal docente, el acompañamiento brindado a los profesores en esta inserción laboral, los planes futuros del proyecto, entre otros.

\section{Recolección de evidencias}

El objetivo de este instrumento se basó en la recolección de datos tangibles sobre el crecimiento profesional de los profesores a lo largo del tiempo laborado en CI-UNA. La recolección de evidencias consistió en recoger los registros de las evaluaciones hechas a los profesores al final de cada ciclo lectivo por parte de los estudiantes y de una de las desarrolladoras, con el fin de medir su crecimiento profesional. La evaluación realizada por los estudiantes consiste en un cuestionario y la realizada por la desarrolladora consistió en una observación de la clase y análisis de los planeamientos. Este instrumento pretendió brindar objetividad y confiabilidad al estudio.

\section{Cuestionario para empleadores}

El objetivo de este instrumento consistía en recolectar información sobre el desempeño laboral desde el punto de vista de los empleadores de los profesores que trabajan o han trabajado en CI-UNA y que laboraron durante el 2016 en otras instituciones. El instrumento está compuesto por seis preguntas abiertas que indagan aspectos como las cualidades de dichos profesores, las responsabilidades y los deberes que estos tienen, y su desempeño laboral global, entre otros. Se aplicó este instrumento a un total de diez empleadores de diferentes instituciones, tales como la Universidad Nacional, Campus Pérez Zeledón y Campus Coto, la Universidad Internacional San Isidro Labrador (UISIL), la Universidad Latina 
Revista Universidad en DiÁlogo • Vol. 10, N. ํ 2, Julio-Diciembre, 2020 • 55-77

ISSN 2215-2849 • EISSN: 2215-4752

URL: https://www.revistas.una.ac.cr/index.php/dialogo/index Correo eleCtrónico: universidadendialogo@una.ac.cr

DOI: https://doi.org/10.15359/udre.10-2.3

de Costa Rica, directores del Ministerio de Educación Pública y empleadores de institutos privados. Dicha información es vital para lograr comprobar el crecimiento que han tenido los profesores al estar trabajando en CI-UNA y en otras empresas o instituciones al mismo tiempo.

\section{Análisis}

Cuando los estudiantes terminan sus carreras y empiezan la búsqueda de empleo no siempre es fácil para ellos. Muchos no tienen ninguna conexión de trabajo y la gran mayoría cuenta con muy poca o nula experiencia laboral. Solo en algunos casos los estudiantes graduados se encuentran con la ayuda de instituciones que les brindan oportunidades de trabajo a profesionales sin experiencia. Tal es el caso del proyecto de extensión de la Universidad Nacional CIUNA. Este proyecto ofrece clases de Inglés a las personas de la comunidad de Pérez Zeledón, en Costa Rica, a la vez que les brinda oportunidades laborales a los estudiantes graduados de la carrera Enseñanza del Inglés de la misma universidad que se encuentran en búsqueda de su primer trabajo. Por este motivo, esta investigación pretende estudiar cómo CI-UNA contribuye en la inmersión laboral de los estudiantes graduados de la carrera Enseñanza del Inglés de la Universidad Nacional, Sede Regional Brunca, Campus Pérez Zeledón.

Después de analizar la información recolectada, se encontró que el $100 \%$ de los profesores de Inglés que fueron parte de este estudio expresan una opinión muy positiva de CI-UNA y de lo que esta institución hace tanto por los profesores que apenas comienzan a laborar como por la comunidad en general. En el cuestionario, la desarrolladora dejó en claro que el fin con el que nació CI-UNA no fue para dar empleo a profesores de Inglés graduados de la Universidad Nacional, pero que con el tiempo se ha vuelto también parte del proyecto. Por otro lado, al preguntarle a los profesores sobre los beneficios que han obtenido al trabajar para CI-UNA, el $71 \%$ de los profesores concordó en que el mayor beneficio ha sido la experiencia laboral tan necesaria para poder crecer como profesionales (ver figura 2).

Los docentes también resaltaron que la experiencia que han ganado les ha ayudado a superar las dificultades que enfrentaron durante las primeras clases que impartieron, ya que su confianza en sí mismos ha crecido considerablemente. Todos los empleadores concordaron en que estos profesores se desenvuelven con mucha confianza en sí mismos, lo que enriquece día con día su experiencia laboral. El desempeño de estos se ha calificado dentro del parámetro de excelente. De acuerdo con la información brindada por uno de los empleadores de la UNA, sobresale el dato que revela sobre uno de los profesores que en el año 2014 se encontraba laborando para CI-UNA y el Departamento de Idiomas de la 
URL: https://www.revistas.una.ac.cr/index.php/dialogo/index

CORREO ELECTRÓNICO: universidadendialogo@una.ac.cr

DOI: https://doi.org/10.15359/udre.10-2.3

UNA simultáneamente y logró ser el profesor mejor calificado de todos los profesores del Campus Pérez Zeledón, rebasando así a los de mayor experiencia.

Figura 2

Beneficios obtenidos al trabajar en CI-UNA. Información tomada del cuestionario aplicado a los profesores de CI-UNA

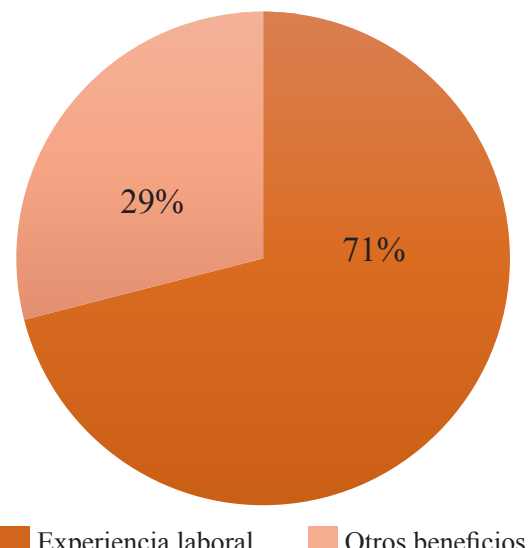

Nota: Información recopilada a través del cuestionario para profesores.

De igual manera, uno de los profesores encuestados, así como una de las desarrolladoras de CI-UNA, expresaron que el hecho de trabajar o haber trabajado para una institución respaldada por una de las universidades más prestigiosas del país, como lo es la Universidad Nacional, les ha ayudado a estos profesores a hacer más atractivo su currículo y así poder encontrar trabajo en otras instituciones. Con respecto a esto, la desarrolladora del proyecto expresó en el cuestionario que "el profesional que se forma aquí ha sido tan exigido a dar lo mejor que no cabe duda de que va a desempeñar un trabajo excepcional en otra institución" (comunicación personal, marzo, 2016) y es precisamente eso lo que los hace atractivos para otros empleadores. Lo anterior es respaldado por los cuestionarios aplicados a los profesores, ya que la mayoría de ellos dijo estar actualmente trabajando también para otras instituciones a pesar de su poca experiencia. Por otro lado, todos los empleadores concuerdan en que los instructores que actualmente laboran para ellos, que han sido egresados de la UNA y que laboran también para CI-UNA, se distinguen por poseer características bastante sobresalientes; entre las características que citan sobresalen que son creativos, proactivos, tienen un alto desempeño y se identifican mucho con el trabajo de aula y con la institución donde laboran. Uno de los empleadores de 
la UNA, Campus Coto, subraya que "el manejo de grupo es una fortaleza de estos profesores, ellos muestran un interés genuino por la docencia, son los más comprometidos y los que se logran visualizar con un concepto mayor de trabajo en equipo" (comunicación personal, marzo, 2016).

Aunado a la experiencia ganada, los profesores también dijeron haber obtenido otros beneficios al trabajar en CI-UNA durante sus primeros años como profesores. Los beneficios más relevantes incluyen el conocimiento adquirido sobre temas como el manejo de grupo, el diseño y la adaptación de actividades y otros recursos didácticos, y la organización, entre otros (ver tabla 2). Adicionalmente, en el cuestionario aplicado a la desarrolladora ella opinó que, además de los beneficios antes mencionados, los profesores de CI-UNA también "pueden aspirar a trabajar en un ambiente bastante pacífico, agradable, desafiante y sobre todo muy enriquecedor" (comunicación personal, marzo, 2016). Otro aspecto relevante es que el $29 \%$ de los profesores consultados admitieron haber empezado sus primeros meses como educadores con muy poca confianza en sí mismos y en su trabajo y que tuvieron algunos problemas tratando de adaptarse a las diferentes situaciones que no fueron previstas cuando estaban estudiando su carrera; algo que ha cambiado conforme ganan más experiencia.

Tabla 2

Otros beneficios obtenidos al trabajar en CI-UNA

\begin{tabular}{lc}
\hline Conocimiento sobre... & Porcentaje de participantes \\
\hline Desarrollo de actividades y otros recursos didácticos & $50 \%$ \\
Manejo de grupo & $57 \%$ \\
Planeamiento & $43 \%$ \\
Organización & $57 \%$ \\
Trabajo con grupos heterogéneos & $43 \%$ \\
\hline
\end{tabular}

Nota. Información tomada del cuestionario aplicado a los profesores de CI-UNA.

Con respecto a lo anterior, todos los docentes expresaron que han mejorado como profesores y como seres humanos de una u otra manera después de trabajar en CIUNA, pues se sienten más confiados en lo que están haciendo y han aprendido a no solo darle importancia a la parte académica, sino también a las relaciones humanas que se construyen con los estudiantes. De igual manera, la desarrolladora asegura que al incorporar a profesores de la misma casa de enseñanza a la que pertenece CI-UNA, el proyecto también se ha visto beneficiado, ya que la preparación que la Universidad Nacional brinda es de calidad reconocida. 
URL: https://www.revistas.una.ac.cr/index.php/dialogo/index

CORREO ELECTRÓNICO: universidadendialogo@una.ac.cr

DOI: https://doi.org/10.15359/udre.10-2.3

En cuanto a cómo han mejorado estos profesores su práctica docente desde su primera clase impartida en CI-UNA hasta su última clase (para la mayoría la última clase tomada en cuenta fue la última del segundo bimestre del 2016), se analizaron los registros de notas de las evaluaciones hechas a los profesores por los estudiantes al final de cada periodo. Dicho análisis muestra que el $65 \%$ de los profesores ha mejorado su práctica docente, pues las notas adquiridas han mejorado; el $29 \%$ se ha mantenido dentro del mismo rango de notas, y solamente el $6 \%$ no ha exhibido una mejora según la evaluación hecha por los estudiantes de CI-UNA. Para ilustrar el mejoramiento en la práctica docente, se analizaron las evaluaciones hechas por los estudiantes a tres profesores (ver figura 3 ).

Juntamente con la evaluación mencionada anteriormente, los docentes también son evaluados cada periodo por una de las desarrolladoras del proyecto CI-UNA. Con respecto a esta evaluación, la desarrolladora encuestada aseguró que "estas visitas no son programadas, es decir, el profesor no está consciente de cuando le corresponde ser observado y evaluado", esto con el fin de que la evaluación sea confiable. Los resultados dejan ver que, según esta apreciación, el $53 \%$ de los profesores muestran una mejora en su desempeño, el $18 \%$ se mantiene dentro del mismo rango de notas y el $29 \%$ no presenta una mejora visible.

Figura 3. Mejora del desempeño de los profesores según los estudiantes. Tres ejemplos que ilustran la mejora en el desempeño de los profesores desde la primera hasta la última clase impartida en CI-UNA.

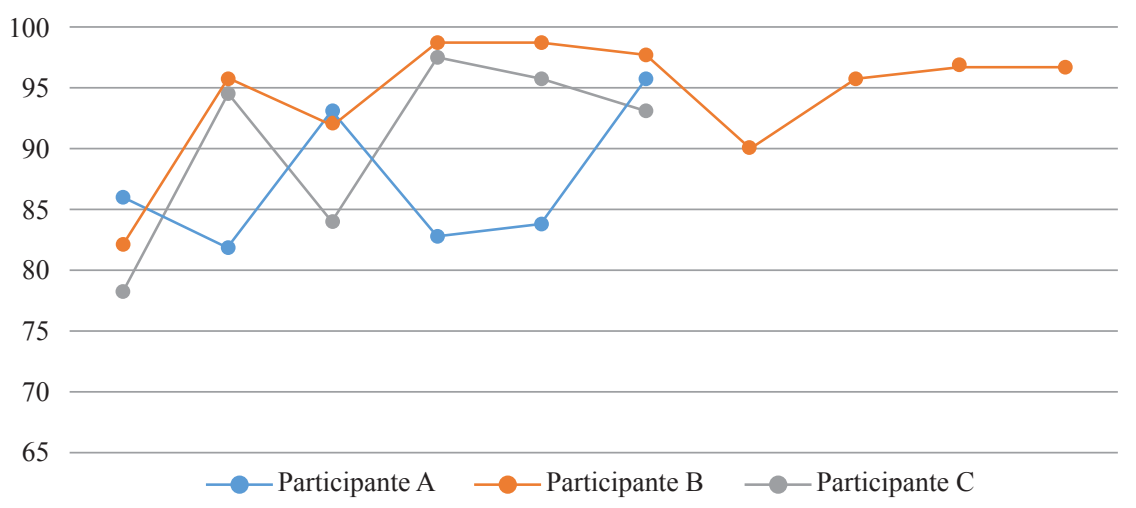

Nota: Información tomada de los registros de las evaluaciones hechas por los estudiantes al final de cada periodo. 
Revista Universidad en DiÁlogo • Vol. 10, N. ํ 2, Julio-Diciembre, 2020 • 55-77

ISSN 2215-2849 • EISSN: 2215-4752

URL: https://www.revistas.una.ac.cr/index.php/dialogo/index CORREO ElECtrónICO: universidadendialogo@una.ac.cr

DOI: https://doi.org/10.15359/udre.10-2.3

La diferencia en los porcentajes obtenidos en ambas evaluaciones puede originarse debido a que los estudiantes tienen la oportunidad de estar en cada una de las clases impartidas y así ser testigos del progreso global del profesor, mientras que la desarrolladora está en el aula solamente en una de las clases, lo que contribuye a que tanto los estudiantes como la desarrolladora perciban de manera diferente el avance del profesor en aspectos relacionados con la pedagogía. Sin embargo, es notable que, de acuerdo con ambas evaluaciones, la mayoría de los profesores han mejorado su práctica docente cuando estuvieron trabajando o desde que están trabajando en CI-UNA.

\section{Conclusiones}

El análisis de los datos permitió a las investigadoras reflexionar acerca de los siguientes aspectos. La primera conclusión evidencia que aun cuando CI-UNA no nació con el objetivo de brindar empleo a los profesores que se graduaban de la carrera Enseñanza del Inglés de la Universidad Nacional, este proyecto se ha convertido en uno de doble propósito al brindar la oportunidad a los profesores que no cuentan con experiencia de integrarse al mundo laboral solo tomando en cuenta su perfil de egresados y, sobre todo, su desempeño como estudiantes. Además, se constató que los profesores tienen una percepción positiva del proyecto gracias a esa oportunidad que se les brindó.

Otra conclusión que se alcanzó a través de este estudio fue el mejoramiento de la hoja de vida de los profesores, que al tener como experiencia previa haber trabajado para una de las instituciones más prestigiosas de la zona, y que a la vez es respaldada por la Universidad Nacional, se vuelven más atractivos para otros empleadores que confían en la formación de estos profesionales. Adicionalmente, se determinó que la carga profesional que CI-UNA asigna a sus profesores es bastante demandante, lo que hace que ellos se esfuercen al máximo para lograr alcanzar el nivel de exigencia esperado.

Sumado a lo anterior, también se concluyó que es evidente el mejoramiento en el rendimiento laboral y el crecimiento personal de los profesores que trabajan o trabajaron en CI-UNA durante sus primeros años de experiencia. También se pudo comprobar que el mayor beneficio que los profesores de CI-UNA han obtenido ha sido precisamente la experiencia laboral. De igual manera, los profesores de CI-UNA han mejorado en aspectos como el manejo de grupo y el planeamiento. Aunque estas eran unas de sus áreas fuertes, con el pasar del tiempo su desempeño en las aulas experimentó un mejoramiento significativo. 
URL: https://www.revistas.una.ac.cr/index.php/dialogo/index

CORREO ELECTRÓNICO: universidadendialogo@una.ac.cr

DOI: https://doi.org/10.15359/udre.10-2.3

Cabe mencionar que la totalidad de los empleadores tienen una opinión muy positiva de los instructores egresados del BEI y de los profesores de CI-UNA. Dentro de los aspectos más relevantes se mencionan su excelente desempeño lingüístico, su esmero por la calidad en el trabajo que desempeñan en las aulas y su alto sentido de pertenencia a la institución para la cual trabajan. Además, estos instructores destacan en el trabajo que realizan en otras empresas por su nivel de cumplimiento, el óptimo manejo de grupo, lo cual se les atribuye como una gran fortaleza, y el trabajo que realizan en equipo.

Finalmente, tal vez la conclusión más significativa de este estudio es que se logró determinar que el proyecto de extensión CI-UNA de la Universidad Nacional es el primer acercamiento que tienen estos profesores, que no poseen experiencia ni un vasto currículo, a una institución pública, lo cual se convierte en una gran oportunidad de trabajo y de crecimiento personal para cada uno de ellos.

\section{Recomendaciones}

Aunque se logró confirmar que los instructores de CI-UNA reciben capacitaciones y acompañamiento profesional de una de las desarrolladoras, se recomienda establecer un mecanismo constante de desarrollo profesional que involucre a especialistas de diversas áreas prioritarias para un mayor desempeño de calidad dentro de las aulas.

También, se sugiere a las desarrolladoras del proyecto continuar con la iniciativa que les ha movido de reclutar, en su mayoría, a egresados del Bachillerato en la Enseñanza del Inglés de la UNA, ya que, con el pasar del tiempo, se vuelve más difícil la rápida inserción de los egresados en el mercado laboral.

Esta iniciativa debería compartirse con la totalidad de los estudiantes del Bachillerato en la Enseñanza del Inglés del Campus desde que inician, para que sea vista como una fuente de motivación durante su permanencia en la carrera; asimismo, darles a conocer que si su desempeño como estudiantes es óptimo tendrían la posibilidad de que, una vez transcurrida su respectiva graduación, ellos logren una plaza en una institución de alto prestigio como lo es CI-UNA.

Debido a que una planta de profesores de CI-UNA sólida y estable se ha constituido a través del tiempo, se recomienda crear una red de instructores sistematizada donde se compartan experiencias positivas y negativas, planeamientos y materiales, con el fin de perpetuar la excelencia de los cursos $\mathrm{y}$, a su vez, potenciar el desarrollo profesional de los profesores. 


\section{Referencias bibliográficas}

Arce, L. (2006). Pérez Zeledón. Heredia: Litografía Morales.

Brenes, C. (13 de mayo, 2012). Centros de inglés con nuevos bríos. El Financiero. Edición 867. http://wvw.elfinancierocr.com/ef archivo/2012/mayo/13/negocios3157718.html

Brown, J. D. (1995). The elements of lenguage curriculum: A sistematic approach to program development. Boston: Heinel and Heinle.

Estatuto Orgánico de la Universidad Nacional. (2015). http://www.sia.una.ac.cr/ index.php?option $=$ com_remository\&Itemid $=76 \&$ func $=$ startdown\&id $=19$

Frank, J. (2012). The role of assessment in language teaching. English Teaching Forum, 3, p. 32. http://files.eric.ed.gov/fulltext/EJ997527.pdf

Gall, M., Gall, J. \& Borg, W. (2003). Educational research: An introduction. USA: Person Education.

Marco Común Europeo de Referencia para las Lenguas. (2002). Marco común europeo de referencia para las lenguas: aprendizaje, enseñanza, evaluación. Instituto Cervantes para la traducción en español.

Nunan, D. (December 9, 2004). Task-based language teaching. Cambridge University Press.

Peacock,M.(1997).TheeffectofauthenticmaterialsonthemotivationofEFLlearners. ELTJournal,51(2),144-156.http://eltj.oxfordjournals.org/content/51/2/144. full.pdf + html? sid=c83d092e-4fde-4357-af41-5577b89aae5d

QS Worldwide university rankings. (2015 - 2016). Top Universities: guides and events. http://www.topuniversities.com/university-rankings/worlduniversity-rankings $/ 2015 \#$ sorting $=$ rank + region $=+$ country $=227+$ facult $\mathrm{y}=+$ stars $=$ false + search $=$

Saunders, M., Lewis, P. \& Thornhill, A. (2007). Research methods for business

Students ( $4^{\text {th }}$ ed.). England: Prentice Hall.

Universidad Nacional. (2005). Modelo pedagógico de la Universidad Nacional. Heredia: Universidad Nacional. 
URL: https://www.revistas.una.ac.cr/index.php/dialogo/index

CORREO ELECTRÓNICO: universidadendialogo@una.ac.cr

DOI: https://doi.org/10.15359/udre.10-2.3

UNA institución para Costa Rica. http://ww.una.ac.cr/index.php/ acerda-de/2012-09-10-20-21-42/2012-09-10-20-25-24w

Universidad Nacional. (2006). Reglamento general sobre los procesos de enseñanza y aprendizaje de la Universidad Nacional. http:// www.defensoria.una.ac.cr/documentos/REGLAMENTO $\% 20$ GENERAL\%20SOBRE\%20LOS\%20PROCESOS\%20DE\%20 ENSENANZA \%20Y\%20APRENDIZAJE\%20DE\%20LA\%20 UNIVERSIDAD\%20NACIONAL.pdf 